\title{
High-sensitivity sequencing reveals multi-organ somatic mosaicism causing DICER1 syndrome
}

\author{
Leanne de Kock ${ }_{1}^{1,2}$ Yu Chang Wang, ${ }^{3}$ Timothée Revil, ${ }^{3}$ Dunarel Badescu, ${ }^{3}$ \\ Barbara Rivera, ${ }^{1,2}$ Nelly Sabbaghian, ${ }^{2}$ Mona Wu, ${ }^{1,2}$ Evan Weber, ${ }^{4}$ Claudio Sandoval, ${ }^{5}$ \\ Saskia M J Hopman, ${ }^{6}$ Johannes H M Merks, ${ }^{6}$ Johanna M van Hagen, ${ }^{7}$ \\ Antonia H M Bouts, ${ }^{8}$ David A Plager, ${ }^{9}$ Aparna Ramasubramanian, ${ }^{9,} 10$ \\ Linus Forsmark, ${ }^{11}$ Kristine L Doyle, $^{12}$ Tonja Toler, $^{13}$ Janine Callahan, $^{14}$ \\ Charlotte Engelenberg, ${ }^{15}$ Dorothée Bouron-Dal Soglio, ${ }^{16}$ John R Priest, ${ }^{17}$ \\ Jiannis Ragoussis, ${ }^{3}$ William D Foulkes ${ }^{1,2,4,18}$
}

- Additional material is published online only. To view please visit the journal online (http://dx.doi.org/10.1136/ jmedgenet-2015-103428)

For numbered affiliations see end of article.

\section{Correspondence to} Dr William D Foulkes, Department of Medical Genetics, Lady Davis Institute, Segal Cancer Centre, Jewish General Hospital, 3755 Cote St. Catherine Road, Montreal, Québec, Canada H3T 1E2; william.foulkes@mcgill.ca

Received 30 July 2015 Revised 7 September 2015 Accepted 18 September 2015 Published Online First 16 October 2015

CrossMark

To cite: de Kock L, Wang YC, Revil T, et al. J Med Genet 2016;53: 43-52.

\section{ABSTRACT}

Background Somatic mosaicism is being increasingly recognised as an important cause of non-Mendelian presentations of hereditary syndromes. A previous wholeexome sequencing study using DNA derived from peripheral blood identified mosaic mutations in DICER 1 in two children with overgrowth and developmental delay as well as more typical phenotypes of germline DICER 1 mutation. However, very-low-frequency mosaicism is difficult to detect, and thus, causal mutations can go unnoticed. Highly sensitive, costeffective approaches are needed to molecularly diagnose these persons. We studied four children with multiple primary tumours known to be associated with the DICER 1 syndrome, but in whom germline DICER 1 mutations were not detected by conventional mutation detection techniques.

Methods and results We observed the same missense mutation within the DICER1 RNase IIIb domain in multiple tumours from different sites in each patient, raising suspicion of somatic mosaicism. We implemented three different targeted-capture technologies, including the novel HaloPlex ${ }^{\mathrm{HS}}$ (Agilent Technologies), followed by deep sequencing, and confirmed that the identified mutations are mosaic in origin in three patients, detectable in $0.24-31 \%$ of sequencing reads in constitutional DNA. The mosaic origin of patient 4's mutation remains to be unequivocally established. We also discovered likely pathogenic second somatic mutations or loss of heterozygosity $(\mathrm{LOH})$ in tumours from all four patients.

Conclusions Mosaic DICER 1 mutations are an important cause of the DICER 1 syndrome in patients with severe phenotypes and often appear to be accompanied by second somatic truncating mutations or $\mathrm{LOH}$ in the associated tumours. Furthermore, the molecular barcode-containing HaloPlex ${ }^{\mathrm{HS}}$ provides the sensitivity required for detection of such low-level mosaic mutations and could have general applicability.

\section{INTRODUCTION}

Mosaicism arises following the acquisition of a de novo mutation during post-zygotic development, resulting in an individual with two populations of cells that are genetically distinct. ${ }^{1}$ Mosaicism is being increasingly recognised as the cause of a diverse range of sporadic albeit likely genetic clinical disorders, the aetiology of which was previously unknown. ${ }^{1-4}$ This is largely attributed to improved genomic sequencing technologies that have provided better ability to detect genetic changes in subpopulations of cells. Despite this, detecting low-level mosaicism is still challenging.

The DICER1 syndrome or pleuropulmonary blastoma (PPB) familial tumour and dysplasia syndrome (OMIM \#601200) is typically caused by heterozygous germline mutations in DICER1, which encodes a small RNA endoribonuclease responsible for processing hairpin precursor miRNAs into mature miRNAs that in turn posttranscriptionally regulate expression of target messenger RNAs. Since studies began on DICER1 in $2009,^{5}>140$ heterozygous germline mutations have been published. ${ }^{6}$ DICER1 syndrome is associated with a predisposition to several rare phenotypes including PPB, cystic nephroma (CN), ovarian Sertoli-Leydig cell tumour (SLCT), multinodular goitre (MNG), nasal chondromesenchymal hamartoma (NCMH), pineoblastoma, pituitary blastoma (PitB) and other rare conditions. ${ }^{6}$ DICER1 syndrome has autosomal-dominant inheritance with variable penetrance and may present from infancy through adolescence and occasionally later. ${ }^{6}$ Using whole-exome sequencing, Klein et $a l^{7}$ have recently reported mosaic missense mutations in DICER1, affecting the RNase IIIb metal-ion binding domain, in peripheral blood DNA from two infants; allele frequencies were $21 \%$ and $28 \%$, respectively. Each infant had extensive bilateral lung cysts consistent radiographically with cystic PPB, developed bilateral Wilms tumour (in one child in large kidneys with underlying renal dysmorphology) and had global developmental delays and various overgrowth stigmata. Klein et al used 'Global delay, Lung cysts, Overgrowth and Wilms (GLOW)' syndrome to describe this phenotype. Brief mention of mosaic DICER1 mutations also appears in one abstract. $^{8}$

Here, we describe our detailed molecular investigation of four children with multiple primary tumours consistent with the DICER1 syndrome 
phenotype, but in whom germline DICER1 mutations had not been detected by Sanger sequencing or multiplex ligation-based probe amplification (MLPA) assay ${ }^{9}$ of genomic DNA isolated from peripheral blood or saliva.

\section{MATERIALS AND METHODS}

Details of sample acquisition, gDNA extractions, Sanger sequencing and the MLPA assay are presented in the online supplementary material methods.

\section{Targeted captures and next-generation sequencing}

We interrogated both tumour and normal tissues for evidence of somatic mosaicism using a standard HaloPlex targeted capture ${ }^{10}$ (Agilent Technologies, Santa Clara, USA), an in-house Fluidigm Access Array (Fluidigm, San Francisco, California, USA), and a novel development of the HaloPlex assay that incorporates molecular barcodes for high-sensitivity sequencing as a custom design (HaloPlex ${ }^{\mathrm{HS}}$ ).

In brief, the HaloPlex ${ }^{\mathrm{HS}}$ targeted capture method is specifically designed to identify low allele frequency variants through the attachment of a 10-nucleotide-long molecular barcode to the captured sample DNA molecules. High sensitivity is supported by the capture of up to eight different restriction fragments per targeted base in the region of interest (figure 1). In our case, $>75 \%$ of the targeted bases were covered by at least four probes (see online supplementary figures S1A-B and S2). During downstream analysis of the sequencing data, molecular barcode sequence data are used to collapse reads originating from the same sample molecule, which improves base calling accuracy and allows for accurate quantification of the mutant allele fraction within each sample as it excludes possible PCR amplification bias. The design used in this study captures $499 \mathrm{~kb}$ and encompasses the full DICER1, DROSHA, AGOII, TRBP2 and DGCR8 loci, all miRNA-processing-associated genes (see online supplementary figure S1). ${ }^{6}{ }^{11}$ We will be pleased to make the design of this array available; please contact the authors for further details. We processed three gDNA samples from different sites from each child for a total of 12 samples (excluding controls), 11 of which were non-tumourous. The sequencing data were generated on the Illumina HiSeq2500 sequencer
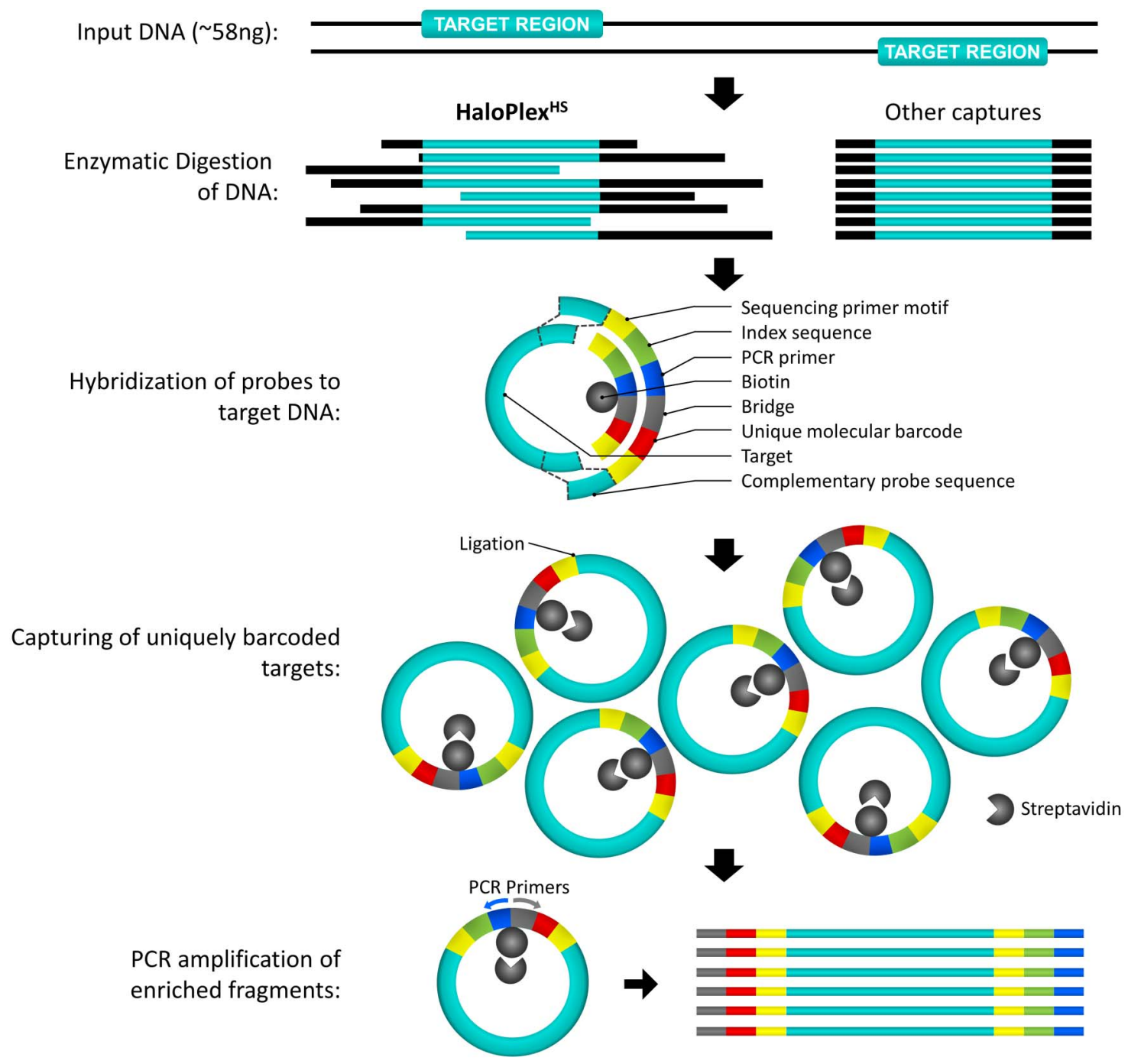

Figure 1 Graphical representation of the HaloPlex ${ }^{\mathrm{HS}}$ design principle. The HaloPlex ${ }^{\mathrm{HS}}$ captures up to eight different restriction fragments per targeted base in the region of interest, ensuring that the great majority of target region is ultimately covered. During hybridisation, each sample is given a unique index sequence (green), allowing for pooling of up to 96 samples per sequencing lane. A degenerate molecular barcode sequence (red) is also incorporated during hybridisation, which makes it possible to track individual target amplicons during sequence analysis and to remove duplicate reads if necessary. 
using the $150 \mathrm{bp}$ paired-end sequencing protocol across four rapid flow-cell lanes. The depth of coverage achieved for each sample is depicted in online supplementary figure S1C and D. We also used the standard HaloPlex targeted capture system with a similar probe design (see online supplementary figure S2), but which does not incorporate molecular barcodes to facilitate the removal of duplicate reads. We sequenced a total of 28 gDNA samples using the Illumina MiSeq sequencer, 17 of which were non-tumourous and 11 were tumour derived. Lastly, we used a custom design Fluidigm Access Array that selectively captures all exons and exon-intron boundaries of DICER1 (described previously) $^{12}$ to allow for cross-platform validation of our findings. Following capture with Fluidigm, we sequenced 22 normal gDNA samples and 15 tumour gDNA samples using the Illumina MiSeq for a total of 37 samples (see online supplementary figure S3).

\section{Inspection of suspected mosaic mutations}

Initial analysis of the suspected mosaic missense mutations in the HaloPlex ${ }^{\mathrm{HS}}$ data was performed using the SureCall software V.3.0 (beta version), provided by Agilent Technologies. Sequencing reads were aligned to the human genome (hg19) and the duplicate reads were removed. The relative frequency of the four bases at the position of each mutation were manually inspected and recorded. We analysed the standard HaloPlex data in the same way using the then publically available SureCall software (V.2.0.7.0) and analysed the Fluidigm-generated data using the Integrative Genomics Viewer software (IGV V.2.3).

\section{Calculation of threshold of detection in the HaloPlex ${ }^{\mathrm{HS}}$ data}

We calculated the percentage of false nucleotides at the position of the respective mosaic DICER1 RNase IIIb mutations in each of the samples by dividing the number of reads containing an aberrant base by the total number of reads, multiplied by 100 (eg, blood DNA from patient 2 contained 4 of 9650 reads with a false $\mathrm{T}$ allele, or $0.04 \%$ ). Using all samples with $>100$ reads covering the region of interest, we calculated the median number of false reads to be $0.04 \%$ (range $0-0.35 \%$ ). By studying the distribution of false positive reads at the positions of the four RNase IIIb mutations, we were able to calculate the total number of false reads. We then took the fourth quintile of this distribution $(0.06 \%)$ as the cut-off, below which we regarded all mutation calls as false positive. To be conservative, we considered the threshold of detection to be $0.1 \%$.

\section{Bioinformatic methods}

The standard HaloPlex and Fluidigm-generated data sets were separately analysed using our custom bioinformatics pipeline as follows: raw paired-end reads were trimmed using Trimmomatic V. $0.33^{13}$ to a minimum length of 30 nucleotides. Illumina Truseq adapters were removed in palindrome mode. A minimum Phred quality score of 30 was required for the $3^{\prime}$ end. Single end reads as well as paired-end reads failing previous minimum quality controls were discarded. Individual read groups were aligned, using bwa V.0.7.12, with default parameters, ${ }^{14}$ to the UCSC hg19 reference human genome from Illumina iGenomes website. ${ }^{15}$ Aligned reads from multiple read groups belonging to the same sample were indexed, sorted and merged using Sambamba V.0.5.4, ${ }^{16}$ a faster implementation of the Samtools algorithms. ${ }^{17} 18$

Various quality control parameters were used, including depth of coverage, based on metrics collected for each sample using bedtools V.2.24.0 ${ }^{19}$ and aggregated using custom Python V.2.7.9 codes. We applied GATK V.3.3.0 base quality score recalibration and indel realignment. ${ }^{20}$ As amplification duplicates were not removed, we also added '-nt NONE' parameter to change the corresponding default down-sampling behaviour.

We performed SNP and INDEL discovery and genotyping across each cohort of samples simultaneously using standard hard filtering parameters according to GATK Best Practices recommendations. ${ }^{21} 22$ In addition to HaplotypeCaller algorithm, we also used UnifiedGenotyper in separate runs. Samtools' new multiallelic calling model (-m parameter), as implemented in bcftools V.1.2, was also used. ${ }^{23}$ All variants were annotated with functional prediction using snpEFF V.3.6. ${ }^{24}$ Additionally, functional annotation of variants found in two public databases (ie, NCBI dbSNP V.142 ${ }^{25}$ and dbNSFP V.2..$^{2627}$ ) were added using SnpSift, as part of the same software package. ${ }^{28}$

HaloPlex ${ }^{\mathrm{HS}}$ data set was analysed using the SureCall software V.3.0 (beta version). All variants obtained from all three data sets were inserted into a Gemini database, ${ }^{29}$ aggregated and selected according to snpEFF predictions. Finally, they were manually validated against read alignments using IGV software V.2.3. ${ }^{30} 31$

\section{Comparison of locus coverage and percentage of homozygosity}

The average coverage of the five targeted loci was calculated using GATK's DepthOfCoverage tool (see online supplementary figure S4). The total number of SNPs per locus was extracted from a Gemini database loaded with only the HaplotypeCaller variants (see above for details). The percentage of homozygosity was then calculated using the number of homozygous alternate or reference SNPs for the DICER1 locus or the other four targeted loci.

\section{RESULTS}

The clinical presentation of each child is outlined in table 1 and figure 2 with further details available in the online supplementary data and figures S5 and S6. The clinical cases of both patients $1^{32}$ and $2^{33}$ have been previously described. The family history for all four children was unremarkable (see online supplementary figure S5).

In most individuals with clinical features suggestive of the DICER1 syndrome, germline truncating mutations in DICER1 are accompanied by specific somatic 'hotspot' missense mutations occurring within the sequence encoding the RNase IIIb domain. ${ }^{6}{ }^{12} 34$ Our initial Sanger sequencing efforts did not identify causal germline mutations in DICER1, but we reasoned that if the tumours contained a somatic DICER1 mutation, the disease presentation could be due to an occult germline mutation in DICER1 or in a closely related gene. We thus undertook Sanger sequencing of gDNA extracted from multiple tumour samples from each patient to determine whether they harboured a somatic DICER1 mutation. We observed the same missense mutation within the sequence encoding the DICER1 RNase IIIb domain in multiple tumours from each patient (patient 1: c.5125G>A [p.D1709N], number of tumours sequenced (n) $=8$; patient 2: c. $5437 \mathrm{G}>\mathrm{C}$ [p.E1813Q], $\mathrm{n}=6$; patient 3 : c.5439G >C [p.E1813D], $\mathrm{n}=5$; and patient 4: c.5425G $>$ A [p. G1809R], n=2) (figure 3). There are a diverse range of missense mutations reported to occur at these particular hotspot locations, ${ }^{6}$ with 215 reports of such hotspot mutations in the literature, 115 of which were confirmed somatic and the great majority of the rest are presumed likely somatic. They mostly affect 1 of 11 nucleotides within the sequence encoding the DICER1 RNase IIIb domain. ${ }^{6}$ We, therefore, thought it very 
Table 1 Clinical summary

\begin{tabular}{|c|c|c|}
\hline Patient & $\begin{array}{l}\text { Age at pathological } \\
\text { diagnosis }\end{array}$ & Disease \\
\hline Patient 1 & $\begin{array}{l}\text { In utero* } \\
\text { Birth*; } 5 \text { days } \\
\text { Birth* } \\
9 \text { months }^{*} ; 1.7 \text { years } \\
25 \text { months } \\
25 \text { months } \\
3.1 \text { years } \\
8 \text { years } \\
10.5 \text { years } \\
10.9 \text { years }\end{array}$ & $\begin{array}{l}\text { Bilateral lung cysts } \\
\text { Bilateral CCAM, revised later to type I PPB } † \\
\text { Scrotal web and testicular cyst } \\
\text { Multiple small bowel polyps: enteritis cystica } \\
\text { profundat } \\
\text { Type II PPB } \dagger \\
\text { PPB brain metastasis \#1 } \dagger \\
\text { PPB brain metastasis \#2 } † \\
\text { NCMH } \\
\text { PPB brain metastasis \#3† } \\
\text { PPB brain metastasis \#4 } \dagger\end{array}$ \\
\hline Patient 2 & $\begin{array}{l}13 \text { months }^{*} ; 1.2 \text { years } \\
13 \text { months }^{*} ; 2.5 \text { years } \\
\text { Birth*; } 2 \text { years } \\
7.7 \text { years } \\
10.6 \text { years } \\
13.4 \text { years } \\
15.1 \text { years } \\
15.7 \text { years } \\
17.2 \text { years }\end{array}$ & $\begin{array}{l}\text { Hamartomatous bilateral renal cysts, NOS } \dagger \\
\text { Benign multifocal bilateral lung cysts, NOS } † \\
\text { L ocular 'pre-phthisical changes': vascular } \\
\text { mass and recurrent retinal detachments } \\
\text { Pineoblastomat } \\
\text { Follicular variant of papillary thyroid } \\
\text { carcinomat } \\
\text { L ovarian SLCT } \dagger \\
\text { Unusual hamartomatous nasal polyp, not } \\
\text { otherwise categorisedt } \\
\text { R ovarian SLCT } \\
\text { R teratoid CBME† }\end{array}$ \\
\hline Patient 3 & $\begin{array}{l}10 \text { days } \\
6 \text { months } \\
1 \text { month }\end{array}$ & $\begin{array}{l}\text { R CCAMt, revised to type I PPB十; L lung cysts } \\
\text { Hamartomatous juvenile intestinal polyps } \\
\mathrm{R} \text { renal medullary malformation with } \\
\text { disorganised collecting system and dilated } \\
\text { lymphatic vesselst } \\
\mathrm{LCN \dagger} \\
\text { NCMH }\end{array}$ \\
\hline Patient 4 & $\begin{array}{l}1.7 \text { years } \\
4.1 \text { years }\end{array}$ & $\begin{array}{l}\text { Bilateral multifocal lung cysts; L CCAMt; } \\
\text { revised to type I PPB } † \\
\text { R infected pulmonary cyst } † \\
\text { L residual type I PPB with polypoid type II } \\
\text { PPB† }\end{array}$ \\
\hline
\end{tabular}

${ }^{*}$ Age at detection.

tPathological diagnosis.

CBME, ciliary body medulloepithelioma; CCAM, congenital cystic adenomatoid malformation; CN, cystic nephroma; L, left; NCMH, nasal chondromesenchymal hamartoma; NOS, not otherwise specified; PPB, pleuropulmonary blastoma; R, right; SLCT, Sertoli-Leydig cell tumour.

unlikely that the same hotspot mutation would occur in all independent disease foci in each child, raising suspicion of somatic mosaicism for the identified DICER1 mutations.

We confirmed a mosaic distribution of the respective mutations in patients $1-3$ using the three targeted-capture platforms: the mutant allele fraction was significantly higher in tumour samples and was detected at low levels in multiple normal tissues from the three patients. The average mutant allele frequency in non-tumour samples ranged from $0 \%$ to $13.58 \%$ in normal tissues from patient 1 (12.77-60.80\% in tumour samples); from $0 \%$ to $1.33 \%$ normal in tissues from patient 2 $(0-99.41 \%$ in tumours) and from $0 \%$ to $31.73 \%$ in normal tissues from patient 3 (6.55-91.89\% in tumours). The numbers of sequencing reads and the percentage of mutant and wildtype alleles per patient sample are indicated in table 2, online supplementary table S1 and figure 4A-D. Comparing the four technologies used, it is clear that the HaloPlex ${ }^{\mathrm{HS}}$ data offers greater precision, given the ability to remove duplicate reads and in so doing increase the base calling accuracy, as described above. We considered $0.1 \%$ to be the threshold for detection, below which all mutant alleles detected were considered false positive (see table 2 and the 'Materials and methods'). Of particular interest is patient 2, c.5437G $>$ C, where in saliva DNA, using HaloPlex ${ }^{\mathrm{HS}}$, we identified 5 of 1972 reads with the $\mathrm{C}$ allele $(0.25 \%)$, whereas only one read was seen for a $\mathrm{T}$ allele at this position $(0.05 \%)$. This $\mathrm{T}$ allele is clearly a false positive read. By contrast, in blood DNA, we identified 4 of 9650 reads $(0.04 \%)$ for both the $\mathrm{C}$ and $\mathrm{T}$ alleles (table 2), suggesting that both the $\mathrm{C}$ and the $\mathrm{T}$ alleles are false positives. From this result, we conclude that the c.5437G $>\mathrm{C}$ mutation is present in saliva DNA, but not in blood DNA. The c.5437G $>$ C mutation was also identified at low levels in urine (table 2).

We hypothesised that, in the setting of a mosaic DICER1 RNase IIIb mutations, we might discover second somatic mutations outside of the RNase IIIb domain, which initiate two-hit tumourigenesis as seen in most DICER1-related tumours. All three data sets were analysed as described in the 'Materials and methods', an outline of which is presented in online supplementary figure S4. We identified individually distinct second somatic likely deleterious DICER1 mutations in patient 2's left ovarian SLCT (c.4626_4626delG; p.Q1542Hfs*18) and sinonasal inflammatory polyp (c.4458_4458delA; p.K1486Nfs*4), in patient 3's NCMH (c.4651_4652insTGCT; p.E1551Vfs*7) and in patient 4's type II PPB, which arose in a pre-existing lung cyst (c.1966C>T; p.R656*) (see online supplementary figure S6A). Each of these second somatic mutations was validated via Sanger sequencing and is predicted to prematurely truncate the DICER1 protein (see online supplementary table S1 and figure 4). Furthermore, we detected loss of heterozygosity $(\mathrm{LOH})$ in one of patient 1's PPB brain metastases and in three additional lesions from patient 2 (follicular thyroid carcinoma, right SLCT and kidney cysts). Evidence of LOH in the tumours is supported by both quantitative and qualitative analyses, as presented in figure 4E-H, online supplementary figure S7 and table S2. In online supplementary figure S7A, LOH is evident in the tumours from patient 1 , column 8 , and in patient 2 , columns 6 and 7, where there is a visible reduction in the average coverage of the DICER1 locus relative to the respective germline samples. Similarly, in online supplementary figure S7B, there is a visible increase in the per cent SNP homozygosity for tumours occurring in patient 1 , column 7 , and patient 2 , columns 5 and 6, which is indicative of $\mathrm{LOH}$.

Despite the time interval between the cancer diagnosis and blood sampling, it is possible that we may be detecting traces of circulating tumour DNA in blood samples or infiltrating tumour cells in normal tissue sampled from areas adjacent to tumours. In addition to collecting tissues that are less likely to contain contaminating tumour DNA (eg, hair and saliva), we also wanted to carry out additional analyses to explicitly determine whether we were indeed picking up contaminating tumour DNA. Three of the above exonic somatic mutations were not detected at all in germline samples in the HaloPlex ${ }^{\mathrm{HS}}$ data set. The remaining somatic mutation found in patient 4's type II PPB was detected at $0.04 \%$ in blood DNA, well below the $0.1 \%$ threshold for likely real mutations (see 'Materials and methods'), and we are thus able to establish that the mosaic mutation-containing alleles detected in the non-tumorous samples were not derived from infiltrating tumour cells or circulating tumour DNA. The identified second somatic mutations and the number of alternate and wildtype alleles per patient sample are summarised in online supplementary table S3.

\section{DISCUSSION}

With the use of a targeted approach, combined with deep and ultra-deep sequencing, we detected low-level DICER1 mutant allele fractions in three patients exhibiting mosaicism for the detected mutations. The fourth case is also likely to be a mosaic 


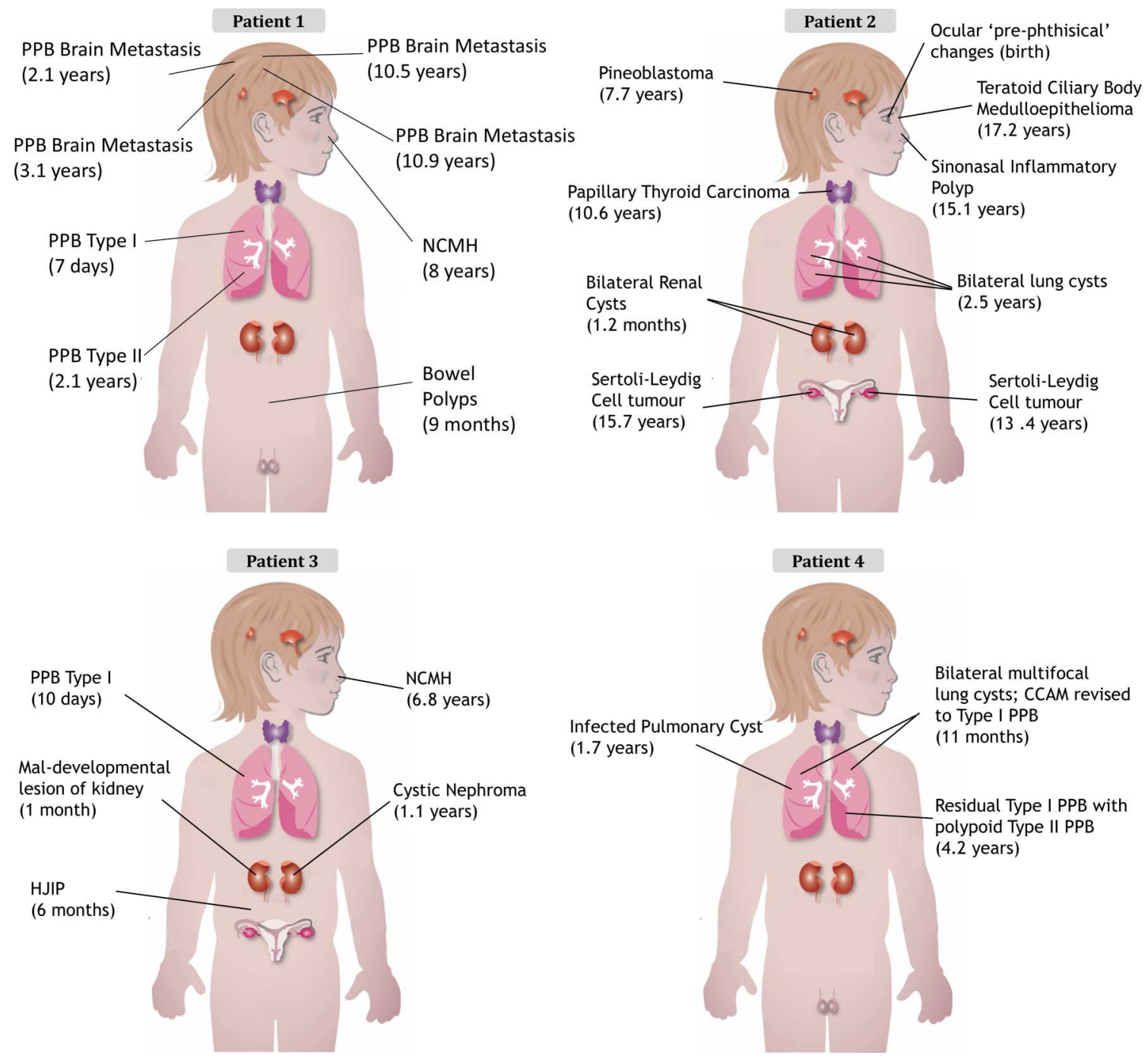

Figure 2 Diagnosis and (age at diagnosis) for each child's tumour. CCAM, congenital cystic adenomatoid malformation; HJIP, hamartomatous juvenile intestinal polyps; NCMH, nasal chondromesenchymal hamartoma; PPB, pleuropulmonary blastoma.

for DICER1. These mosaic missense mutations were localised to 'hotspots' within the sequence encoding the DICER1 RNase IIIb domain and have been shown to selectively reduce $5 \mathrm{p}$ miRNA processing. ${ }^{34-36}$ We also discovered likely pathogenic second somatic mutations or $\mathrm{LOH}$ in tumours from all four patients, thus showing that the two-hit model applied to the tumours we studied (table 2 and online supplementary table S1).

The exact developmental stage at which the mosaic mutations were acquired has not been accurately determined, but given the presence of the mutant allele in tissue samples from all three germ layers, we suspect that the mutations occurred prior to gastrulation. ${ }^{1} 37$ The mosaic origin of patient 4's mutation remains to be unequivocally established (table 2, online supplementary table S1 and figures 3 and 4). Without additional normal samples from both the lung(s) and other distant normal sites, we were not able to determine whether (a) the child is a somatic mosaic with an undefined, yet limited distribution of the mutation; (b) mosaicism is present but is confined to the lungs; or (c) the two lung lesions separately acquired the c. $5425 \mathrm{G}>\mathrm{A}$ 'hit' by chance. In the latter case, the $6.9 \%$ mutant allele frequency in the reactive lung tissue would have to be attributed to cancer cells that were not obviously present on detailed histopathological examination (see online supplementary figure S8), and therefore, we do not favour this explanation. Moreover, the detection of a second somatic truncating mutation in the PPB type II sample (see online supplementary table S1) and the absence of any further extrapulmonary DICER1-related lesions in this person support the hypothesis that somatic mosaicism is present but is confined to the lungs. In this case, acquisition of the missense mutation would have occurred much later during embryonic development than in the other three cases.

Of note, mosaic DICER 1 mutations in our cases and the two previously described cases ${ }^{7}$ are localised to the sequence encoding the RNase IIIb domain. We have also identified additional likely pathogenic second somatic mutations or $\mathrm{LOH}$ in the tumours. These findings strongly suggest that the molecular paradigm of multi-organ mosaic RNase IIIb mutations followed by second 'hits' in other regions of DICER1 is precisely the reverse of typical, now well-described DICER1 molecular events in which somatic RNase IIIb mutations follow inactivating germline mutations. ${ }^{6}$ This mosaic paradigm affecting the highly critical RNase IIIb residues may explain the apparently severe phenotype seen in three of our cases as well as the severity of Klein's GLOW syndrome cases and their overgrowth and 
A Patient 1: c.5125G>A

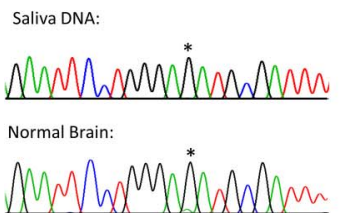

Normal Skin:

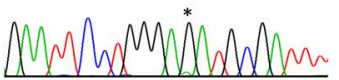

Skin Fibroblasts (Cultured):

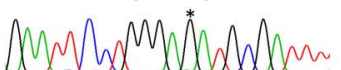

Type I PPB:

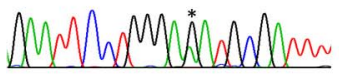

Intestinal polyps:

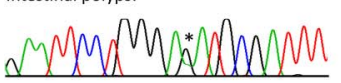

Type II PPB:

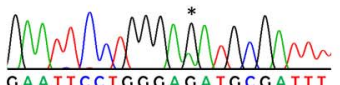

B Patient 2:c.5437G>C C Patient 3: c.5439G $>$ C

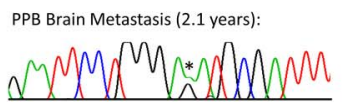

PPB Brain Metastasis (3.1 years):

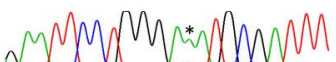

NCMH:

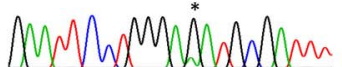

PPB Brain Metastasis (10.5 years):

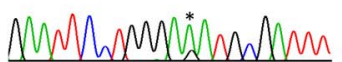

PPB Brain Metastasis (10.9 years):
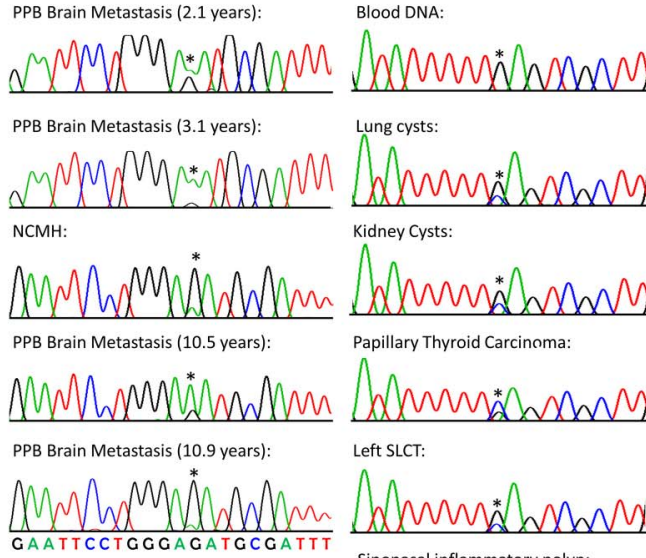

Kidney Cysts:

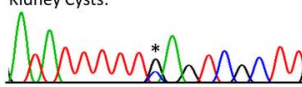

Papillary Thyroid Carcinoma:

A

Left SLCT:

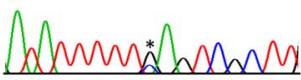

sinonasal inflammatory polyp:

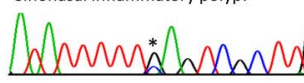

Right SLCT:

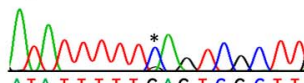

Whminarw
Blood DNA:

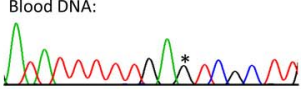

Type I PPB:

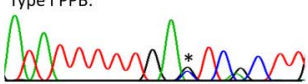

Right normal kidney:

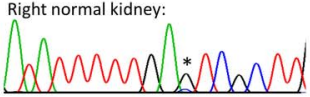

Mal-developmental Right Kidney Lesion:

D Patient 4: c.5425G>A

Blood DNA:

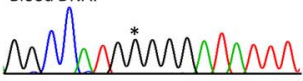

Right Lung Cyst:

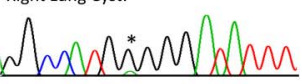

Type II PPB:

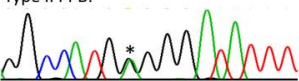

GGCCATGGGGGATATT T

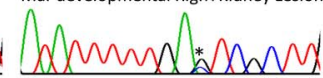

HJIP:

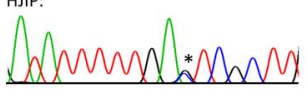

Cysitc Nephroma:

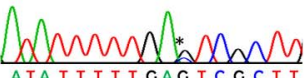

Figure 3 Chromatograms showing the mosaic DICER1 mutation (indicated by an asterisk) in multiple tissue samples. (A) Patient 1 (c.5125G $>A$ ), (B) patient 2 (c.5437G >C), (C) patient 3 (c.5439G >C) and (D) patient 4 (c.5425G>A). HJIP, hamartomatous juvenile intestinal polyps; NCMH, nasal chondromesenchymal hamartoma; PPB, pleuropulmonary blastoma; SLCT, Sertoli-Leydig cell tumour.

developmental delay. Although having several diseases, our cases manifested typical DICER1 phenotypes, and none had overgrowth or developmental delay. One of Klein's cases had renal dysmorphology, and patient 3 had both $\mathrm{CN}$ and contralateral microscopic renal medullary maldevelopment characterised by increased loose mesenchyme, disorganised collecting system and dilated lymphatic vessels, which have not been previously described (see online supplementary data). Both of Klein's patients developed bilateral Wilms tumour; unilateral Wilms and bilateral disease in paired organs are a known feature of DICER1 syndrome. ${ }^{38-41}$ Rather than comprising a new syndrome, we are inclined to believe that multi-organ mosaic RNase IIIb mutations result in an unusually severe overall DICER1 phenotype, within which the pleiotropy typical of DICER1 disease may occasionally result in overgrowth or developmental delay. Identification and analysis of additional mosaic cases may clarify this ambiguity.

Non-RNase IIIb mosaic mutations are likely to exist, and we predict that the phenotype caused by non-RNase IIIb mosaic mutations would be less severe than those caused by mosaic mutations directly affecting the DICER1 RNase IIIb domain, and therefore, such mutations may be more likely to go undetected. There has been one additional reported instance of a de novo germline DICER1 mutation (c.5125G $>\mathrm{C}$; p. $\mathrm{G} 1709 \mathrm{H}$ ) affecting a metal-ion binding residue within the RNase IIIb domain. ${ }^{12}$ The child is severely affected: he presented at birth with a PitB, extensive multifocal bilateral lung cysts and bilateral renal cystic masses. The c.5125G $>$ C mutation was seemingly heterozygous in lymphocyte gDNA, but extensive investigations to confirm or rule out mosaicism were not possible. ${ }^{12}$ The inference from these data is that both mosaic and non-mosaic germline missense mutations affecting exons encoding the metal-ion binding domain of DICER1 underpin a particularly severe disease phenotype and may induce a large number of disease foci per child, depending on the specific tissue distribution of the mutation (patient 4 might exemplify this more limited yet significant mutation distribution). In support of this, we recently identified a paternally inherited novel heterozygous germline DICER1 mutation, c.5441C > T ( $\mathrm{p}$. S1814L), in a girl who developed an SLCT and MNG before the age of 13 years (Wu et al, unpublished data). This mutation, although located within the RNase IIIb domain, does not directly affect one of the critical catalytic or metal-binding residues within this domain (eg, residues 1705, 1709, 1810 and $1813^{42} 43$ ). The less severe phenotype exhibited by this child may possibly be related to the 'sparing' of the above-mentioned metal-ion binding residues. It is notable that no inherited germline DICER1 mutations at a nucleotide encoding a metal-ion binding residue have been reported.

Cancer susceptibility syndromes such as familial adenomatous polyposis (FAP) and the neurofibromatoses (NF) are also associated with a mosaic origin of the causative mutations. In these conditions, the disease course of the mosaic form is reported to be milder than the inherited, non-mosaic presentation. ${ }^{44-46}$ For children with mosaic DICER1 mutations affecting the RNase IIIb domain, the disease appears to be more severe (including earlier onset, greater number of disease foci and greater range of phenotypes) than in the more typical autosomal-dominant forms. This disparity may be attributed to the nature of the mutations required to initiate tumourigenesis in DICER1 syndrome-typically a first-hit truncating germline mutation occurs in any protein-encoding region and a second 'hit' specifically affects the RNase IIIb domain. Such combinations are likely to be rare since it appears the selected second hit nearly always affects a very limited number of nucleotides encoding the RNase IIIb metal ion-binding domains. In contrast, in the DICER1 mosaicism reported here, the initial 'hit' is the acquisition of a missense RNase IIIb hotspot mutation. The second likely truncating mutation occurs anywhere across the gene (see online supplementary table S1), and is therefore, stochastically more likely to occur than a RNase IIIb mutation. Thus, we postulate that the combination of the specific effects of the RNase IIIb mutation and widespread inactivating second hits accounts for more severe clinical manifestations in these children. 
Table 2 Number of reads containing mutant versus wildtype base at position of interest

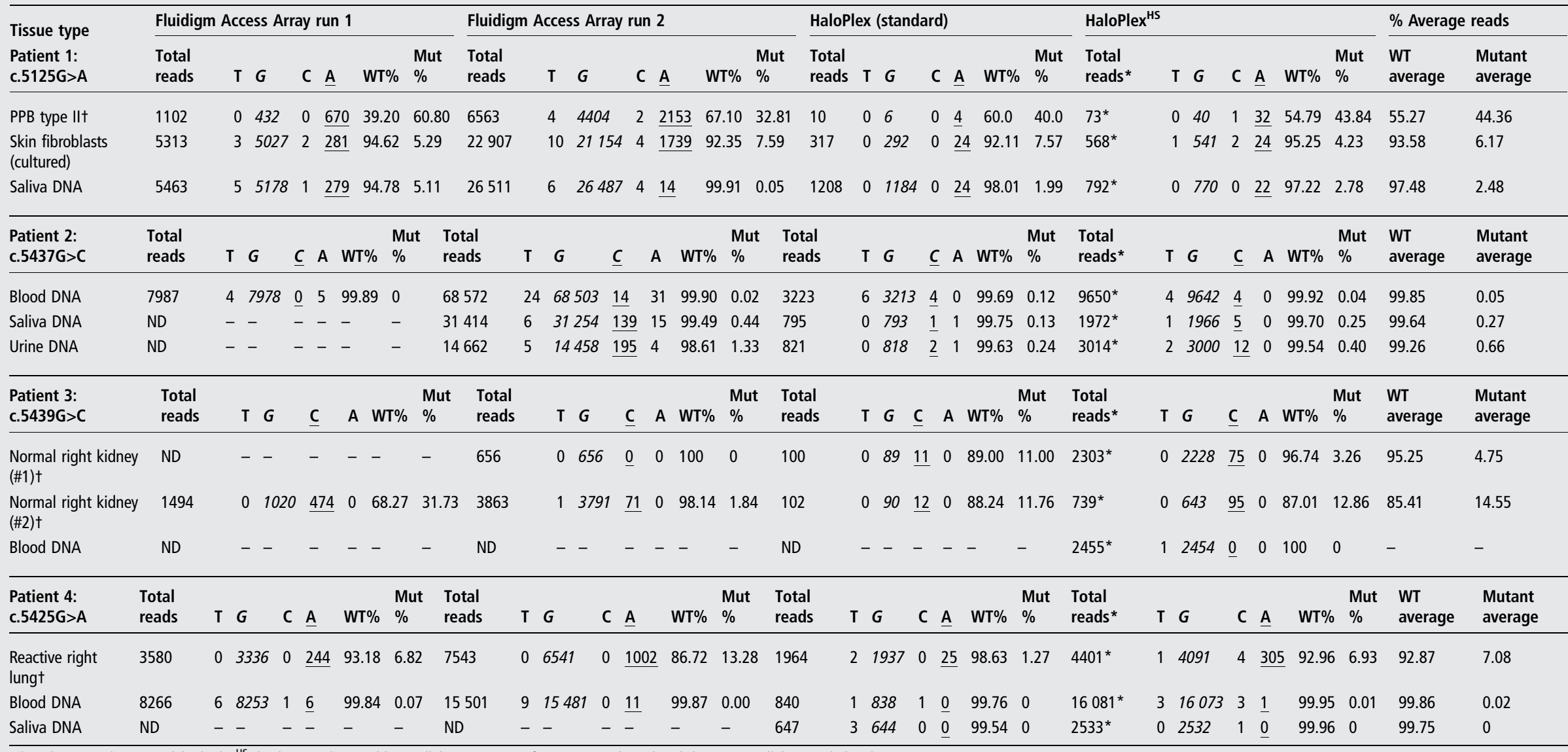

*Duplicate reads removed (HaloPlex ${ }^{\mathrm{HS}}$ data); -, no data. Wildtype allele at position of interest is italicised and the mutant allele is underlined.

tFormalin-fixed paraffin-embedded samples.

Mut, mutant; ND, not done; PPB, pleuropulmonary blastoma, WT, wildtype. 

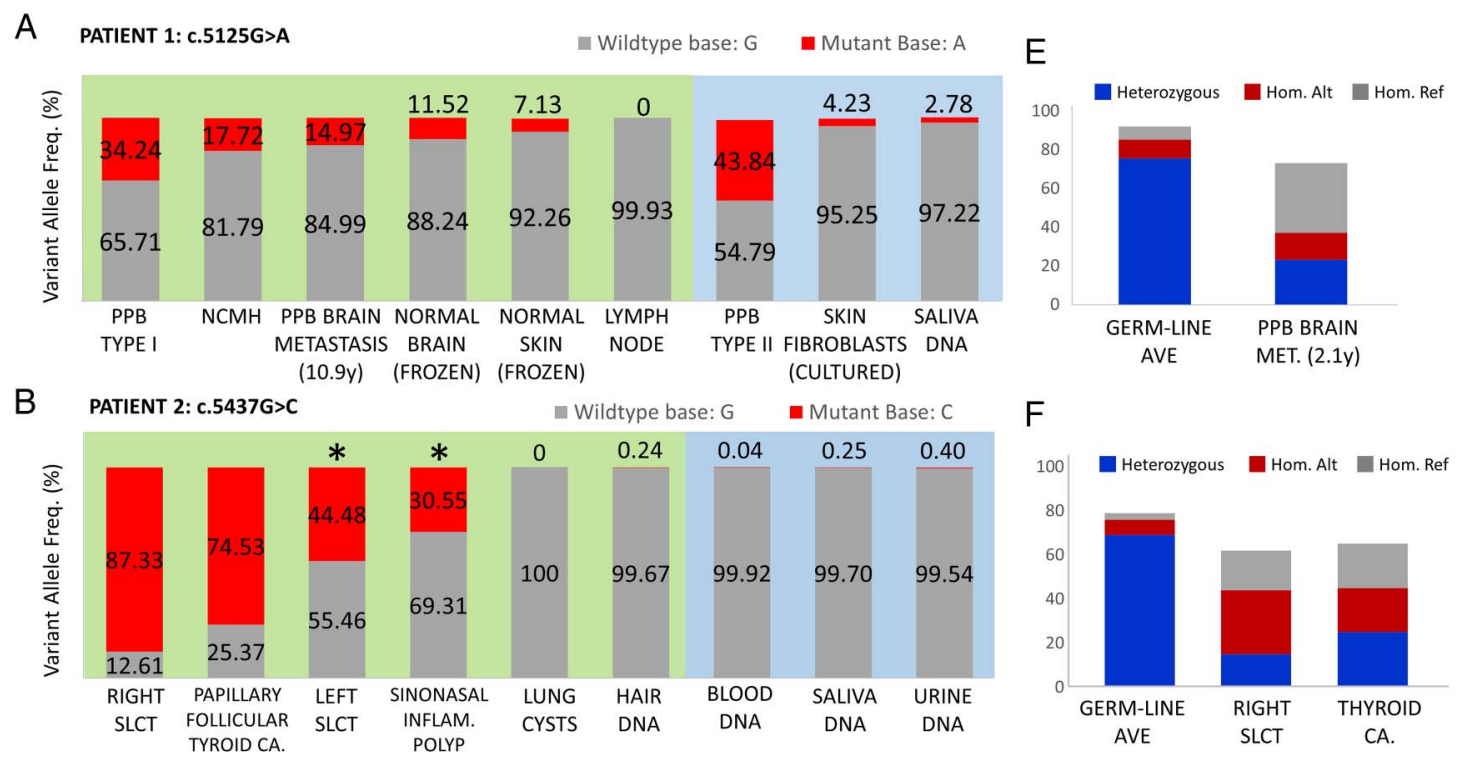

C PATIENT 3: c.5439G $>C$

Wildtype base: $\mathrm{G} \quad$ Mutant Base: C
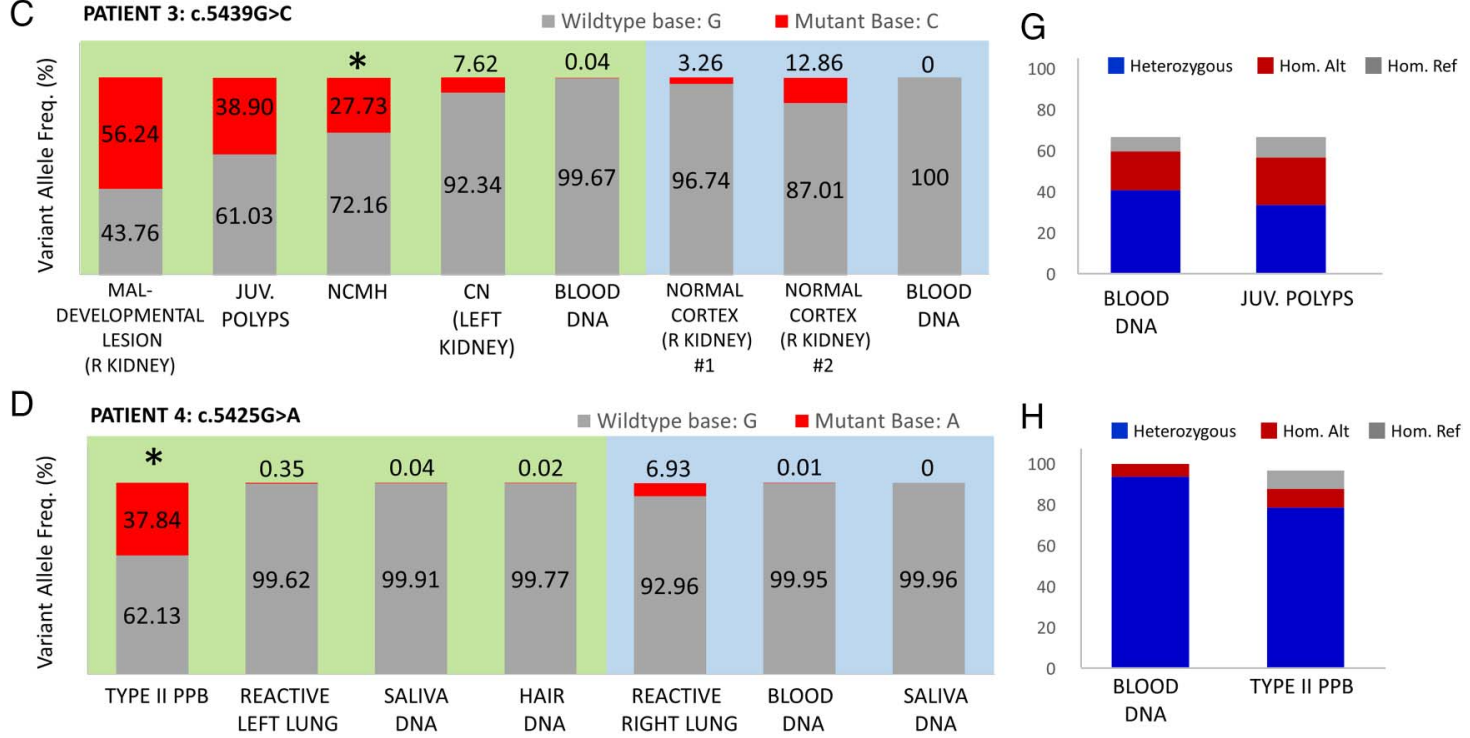

Figure 4 Mutant allele frequencies, second somatic DICER1 mutations and loss of heterozygosity (LOH). Bar graph indicating the mutant allele fraction detected in multiple tissues from (A) patient 1, (B) patient 2, (C) patient 3 and (D) patient 4 . The percentage mutant base fraction (red fill) to wildtype base fraction (grey fill) at the position of interest is indicated for each sample. Green background shading (Left) indicates samples processed using the HaloPlex Standard or Fluidigm Access Array, and blue shading (Right) indicates samples processed using the HaloPlex ${ }^{\mathrm{HS}}$ technology. The percentages given for samples sequenced using the HaloPlex Standard or Fluidigm Access Array are averages of all successful runs. Samples designated with an asterisk are tumours found to carry second somatic, likely truncating DICER1 mutations (see online supplementary table $\mathrm{S} 1$ for details). (E-H) Bar graphs illustrating evidence of $\mathrm{LOH}$ in tumour samples from patient 1 (E) and patient 2 (F) and lack of $\mathrm{LOH}$ in a representative tumour from patient $3(\mathrm{G})$ and patient $4(\mathrm{H})$. In $(\mathrm{E})$ and $(\mathrm{F})$, there is a notable increase in the number of heterozygous to homozygous SNPs in the tumour samples relative to the germline, which is indicative of $\mathrm{LOH}$. This shift is not evident in tumours from patient 3 (G) and patient 4 (H). CN, cystic nephroma; NCMH, nasal chondromesenchymal hamartoma; PPB, pleuropulmonary blastoma; SLCT, Sertoli-Leydig cell tumour.

Therefore, we predict that non-RNase IIIb mosaic DICER1 mutations, like mosaic mutations in FAP and NF, will cause a disease phenotype that is milder than both the autosomaldominant form and that caused by RNase IIIb mosaic DICER1 mutations.

The importance of identifying the causative mutations in these children is several fold: unaffected parents who have an affected child may want to know the risk of recurrence in future pregnancies. Furthermore, the affected children themselves may want to know the probability of transmission to future offspring. Understanding the genetic cause and the mechanism underlying the phenotype provides information that can be used to ascertain such risk. Even in heterozygous germline DICER1 mutation carriers, screening for DICER1-related conditions is problematic, as discussed elsewhere. ${ }^{6}$ Mosaicism further complicates such considerations because the distribution of a somatic mutation would be difficult to determine, but it should be borne in mind that these children may be at increased risk compared with other DICER1 mutation-positive children. In the cases reported here, transmission of the RNase IIIb mutations to the next generation seems unlikely, although in the future, testing of sperm or ova or fetal genetic testing might be considered.

Several high-sensitivity sequencing methods are currently being applied to discover low-frequency mutations, each with its 
own advantages and disadvantages. The most prominent so far are the PCR-based Safe-SeqS ${ }^{47}$ and the Molecular Inversion Probe-based smMIP method. ${ }^{48}$ Whole-genome sequencing with higher than average depth of coverage has also been used to identify de novo mutations that occurred post-zygotically, as was recently reported by Acuna-Hidalgo et al. ${ }^{49}$ Despite these technical advances, detecting low-level mosaicism is still challenging. Low-level mosaic mutations fall below the threshold of sensitivity for many sequencing methods, and other more sensitive technologies are costly and, therefore, may not be practical in either the research or clinical setting. In our hands, the novel HaloPlex ${ }^{\mathrm{HS}}$ target enrichment system containing molecular barcodes provided the sensitivity required for detection of mutant allele fractions as low as $0.24 \%$. We found the HaloPlex ${ }^{\mathrm{HS}}$ to be an economically feasible platform. It is suitable for covering entire genomic regions (in our case, $499 \mathrm{~kb}$ ), but currently $5 \mathrm{Mbp}$ is possible, which is in contrast to PCR product and Molecular Inversion Probe-based methods. Additional advantages of HaloPlex ${ }^{\mathrm{HS}}$ over the smMIP method include a much lower DNA input requirement ( $\sim 50 \mathrm{ng})$ and the redundancy in the HaloPlex ${ }^{\mathrm{HS}}$ probe design allows for the vast majority of targeted bases to be covered by at least four probes, ensuring high coverage, but without increasing the cost of the capture. HaloPlex ${ }^{\mathrm{HS}}$ is likely to be broadly applicable to other situations where mosaicism can occur but yet remain undetected by currently available technologies. The design implemented can be easily adopted by other investigators interested in identifying mutations in DICER1 and other genes encoding the components of the miRNA processing machinery. We also demonstrate the utility of the HaloPlex ${ }^{\mathrm{HS}}$ in FFPE-derived DNA. Our findings suggest that the targeted ultra-deep next-generation sequencing of the DICER1 locus is a useful technique for the identification of mosaic DICER1 mutations.

In summary, by using a new high-sensitivity mutation detection system, we demonstrate that mosaic DICER1 RNase IIIb missense mutations are an occasional and important genetic cause of the DICER1 syndrome in patients presenting with multiple primary tumours associated with the syndrome, but for tumour initiation, they often appear to be accompanied by second somatic truncating non-RNase IIIb DICER1 mutations or $\mathrm{LOH}$.

\author{
Author affiliations \\ ${ }^{1}$ Department of Human Genetics, McGill University, Montréal, Québec, Canada \\ ${ }^{2}$ Lady Davis Institute, Segal Cancer Centre, Jewish General Hospital, Montréal, \\ Québec, Canada \\ ${ }^{3}$ Department of Human Genetics, McGill University and Genome Quebec Innovation \\ Centre, McGill University, Montréal, Québec, Canada \\ ${ }^{4}$ Department of Medical Genetics, Research Institute of the McGill University Health \\ Centre, Montréal, Québec, Canada \\ ${ }^{5}$ Department of Pediatrics, New York Medical College and Maria Fareri Children's \\ Hospital, Valhalla, New York, USA \\ ${ }^{6}$ Department of Pediatric Oncology, Emma Children's Hospital, Academic Medical \\ Center, Amsterdam Zuidoost, The Netherlands \\ ${ }^{7}$ Department of Clinical Genetics, VU University Medical Center, Amsterdam, The \\ Netherlands \\ ${ }^{8}$ Department of Pediatric Nephrology, Emma Children's Hospital, Academic Medical \\ Center, Amsterdam, The Netherlands \\ ${ }^{9}$ Glick Eye Institute, Indiana University School of Medicine, Indianapolis, Indiana, \\ USA \\ ${ }^{10}$ Department of Ophthalmology, University of Louisville, Kentucky, USA \\ ${ }^{11}$ Agilent Technologies, Santa Clara, California, USA \\ ${ }^{12}$ North Hills, California, USA \\ ${ }^{13}$ Petersburg, Indiana, USA \\ ${ }^{14}$ Mahopac, New York, USA \\ ${ }^{15}$ Amsterdam, The Netherlands \\ ${ }^{16}$ Department of Pathology, CHU-Sainte Justine and University of Montreal, \\ Montréal, Québec, Canada
}

${ }^{17}$ Minneapolis, Minnesota, USA
${ }^{18}$ Program in Cancer Genetics, Department of Oncology and Human Genetics, McGill
University, Montréal, Québec, Canada

Acknowledgements The authors dedicate this publication to the memory of Sean Callahan. We thank Charmian Cher, Josh Zhiyong Wang and Martin Angers (Agilent Technologies) for their assistance with the HaloPlex ${ }^{\mathrm{HS}}$ design and capture and Dr Elizabeth Perlman and Dr Michael McDermott for their assistance with pathology review.

Contributors LdK performed the DICER1 hotspot sequencing, initial Fluidigm data analysis and mutation validation and wrote the manuscript. LdK, YCW and BR performed the HaloPlexHS capture. TR and DB performed computational analysis. NS performed the initial constitutional gDNA sequencing and MLPA analysis. MW sequenced the tumours of patient 3 and provided some concepts used in the discussion. EW conducted sample acquisition and associated administration work. CS, SMJH, JHMM, JMvH, AHMB, DAP, AR, KLD, TT, JC and CE referred patients, provided samples and collected clinical information. LF created the design of the HaloPlexHS. DB-DS provided expert pathology opinion. JRP reviewed the diagnostic images, collected clinical information and edited the manuscript. JR and WDF designed the study and edited the manuscript. All authors read and approved the final manuscript.

Funding This research was made possible thanks to the support of the Alex's Lemonade Stand Foundation grant to WDF, the Genome Canada Science Technology Innovation Centre, the Compute Canada Resource Allocation Project wst-164-ab and the Genome Innovation Node grants to JR, and the Vanier Canada Graduate Scholarship to LdK.

Competing interests LF is an employee of Agilent Technologies.

Patient consent Depending on the ages of the participants at the time of recruitment, eligible relatives signed a consent form in accordance with the IRB protocol or participants themselves provided written informed consent.

Ethics approval The study was approved by the Institutional Review Board of the Faculty of Medicine of McGill University, Montreal, Quebec, Canada (no. A12-M117-11A).

Provenance and peer review Not commissioned; externally peer reviewed.

Data sharing statement Sequencing data are available upon request. Please contact the corresponding author.

\section{REFERENCES}

1 Biesecker LG, Spinner NB. A genomic view of mosaicism and human disease. Nat Rev Genet 2013;14:307-20.

2 Frank SA. Somatic mosaicism and disease. Curr Biol 2014;24:R577-81.

3 Lindhurst MJ, Sapp JC, Teer JK, Johnston JJ, Finn EM, Peters K, Turner J, Cannons JL, Bick D, Blakemore L, Blumhorst C, Brockmann K, Calder P, Cherman N, Deardorff MA, Everman DB, Golas G, Greenstein RM, Kato BM, Keppler-Noreuil KM, Kuznetsov SA, Miyamoto RT, Newman K, Ng D, O'Brien K, Rothenberg S, Schwartzentruber DJ, Singhal V, Tirabosco R, Upton J, Wientroub S, Zackai EH, Hoag K, Whitewood-Neal T, Robey PG, Schwartzberg PL, Darling TN, Tosi LL, Mullikin JC, Biesecker LG. A mosaic activating mutation in AKT1 associated with the Proteus syndrome. N Engl J Med 2011;365:611-19.

4 Lupski JR. Genetics. Genome mosaicism—one human, multiple genomes. Science 2013:341:358-9.

5 Hill DA, Ivanovich J, Priest JR, Gurnett CA, Dehner LP, Desruisseau D, Jarzembowsk JA, Wikenheiser-Brokamp KA, Suarez BK, Whelan AJ, Williams G, Bracamontes D, Messinger Y, Goodfellow PJ. DICER1 mutations in familial pleuropulmonary blastoma. Science 2009;325:965

6 Foulkes WD, Priest JR, Duchaine TF. DICER1: mutations, microRNAs and mechanisms. Nat Rev Cancer 2014;14:662-72.

7 Klein S, Lee H, Ghahremani S, Kempert P, Ischander M, Teitell MA, Nelson SF, Martinez-Agosto JA. Expanding the phenotype of mutations in DICER1: mosaic missense mutations in the RNase IIIb domain of DICER1 cause GLOW syndrome. J Med Genet 2014;51:294-302.

8 Doros LA, Turner J, Williams GM, Rossi C, Schultz KAP, Stewart D, Dehner LP, Messinger YH, Hill DA. The DICER1 syndrome: genotype-phenotype correlation in PPB patients. J Clin Oncol 2015. ASCO Annual Meeting (May 29 - June 2, 2015). Vol 33, No 15_suppl (May 20 Supplement), 2015:10022.

9 Sabbaghian N, Srivastava A, Hamel N, Plourde F, Gajtko-Metera M, Niedziela M, Foulkes WD. Germ-line deletion in DICER1 revealed by a novel MLPA assay using synthetic oligonucleotides. Eur J Hum Genet 2014;22:564-7.

10 Mertes F, Elsharawy A, Sauer S, van Helvoort JM, van der Zaag PJ, Franke A, Nilsson $\mathrm{M}$, Lehrach $\mathrm{H}$, Brookes AJ. Targeted enrichment of genomic DNA regions for next-generation sequencing. Brief Funct Genomics 2011;10:374-86.

11 Bahubeshi A, Tischkowitz M, Foulkes WD. miRNA processing and human cancer: DICER1 cuts the mustard. Sci Trans/ Med 2011;3:111ps46. 
12 de Kock L, Sabbaghian N, Plourde F, Srivastava A, Weber E, Bouron-Dal Soglio D, Hamel N, Choi JH, Park S-H, Deal CL, Kelsey MM, Dishop MK, Esbenshade A, Kuttesch JF, Jacques TS, Perry A, Leichter H, Maeder P, Brundler M-A, Warner J, Neal J, Zacharin M, Korbonits M, Cole T, Traunecker H, McLean TW, Rotondo F, Lepage P, Albrecht S, Horvath E, Kovacs K, Priest JR, Foulkes WD. Pituitary blastoma: a pathognomonic feature of germ-line DICER1 mutations. Acta Neuropathol 2014;128:111-22.

13 Bolger AM, Lohse M, Usadel B. Trimmomatic: a flexible trimmer for Illumina sequence data. Bioinformatics 2014:30:2114-20.

14 Li H, Durbin R. Fast and accurate short read alignment with Burrows-Wheeler transform. Bioinformatics 2009;25:1754-60.

15 Illumina. igenomes, ready-to-use reference sequences and annotations. http:// support.illumina.com/sequencing/sequencing_software/igenome.html.

16 Tarasov A, Vilella AJ, Cuppen E, Nijman IJ, Prins P. Sambamba: fast processing of NGS alignment formats. Bioinformatics 2015;31:2032-4.

17 Li H. A statistical framework for SNP calling, mutation discovery, association mapping and population genetical parameter estimation from sequencing data. Bioinformatics 2011;27:2987-93.

18 Li H, Handsaker B, Wysoker A, Fennell T, Ruan J, Homer N, Marth G, Abecasis G, Durbin R. The Sequence Alignment/Map format and SAMtools. Bioinformatics 2009;25:2078-9.

19 Quinlan AR, Hall IM. BEDTools: a flexible suite of utilities for comparing genomic features. Bioinformatics 2010;26:841-2.

20 McKenna A, Hanna M, Banks E, Sivachenko A, Cibulskis K, Kernytsky A, Garimella K, Altshuler D, Gabriel S, Daly M, DePristo MA. The Genome Analysis Toolkit: a MapReduce framework for analyzing next-generation DNA sequencing data. Genome Res 2010:20:1297-303.

21 DePristo MA, Banks E, Poplin R, Garimella KV, Maguire JR, Hartl C, Philippakis AA, del Angel G, Rivas MA, Hanna M, McKenna A, Fennell TJ, Kernytsky AM, Sivachenko AY, Cibulskis K, Gabriel SB, Altshuler D, Daly MJ. A framework for variation discovery and genotyping using next-generation DNA sequencing data. Nat Genet 2011:43:491-8.

22 Van der Auwera GA, Carneiro MO, Hartl C, Poplin R, Del Angel G, Levy-Moonshine A, Jordan T, Shakir K, Roazen D, Thibault J, Banks E, Garimella KV, Altshuler D, Gabriel S, DePristo MA. From FastQ data to high confidence variant calls: the Genome Analysis Toolkit best practices pipeline. Curr Protoc Bioinformatics 2013;11:11.10.11-33.

23 Durbin $\mathrm{R}$, Danecek $\mathrm{P}$, Schiffels S. Multiallelic calling model in bcftools (-m). June 2014. http://samtools.github.io/bcttools/call-m.pdf

24 Cingolani P, Platts A, Wang le L, Coon M, Nguyen T, Wang L, Land SJ, Lu X, Ruden $\mathrm{DM}$. A program for annotating and predicting the effects of single nucleotide polymorphisms, SnpEff: SNPs in the genome of Drosophila melanogaster strain w1118; iso-2; iso-3. Fly (Austin) 2012;6:80-92.

25 Sherry ST, Ward MH, Kholodov M, Baker J, Phan L, Smigielski EM, Sirotkin K. dbSNP: the NCBI database of genetic variation. Nucleic Acids Res 2001;29:308-11.

26 Liu X, Jian X, Boerwinkle E. dbNSFP: a lightweight database of human nonsynonymous SNPs and their functional predictions. Hum Mutat 2011;32:894-9.

27 Liu X, Jian X, Boerwinkle E. dbNSFP v2.0: a database of human non-synonymous SNVs and their functional predictions and annotations. Hum Mutat 2013;34: E2393-2402.

28 Cingolani P, Patel VM, Coon M, Nguyen T, Land SJ, Ruden DM, Lu X. Using Drosophila melanogaster as a Model for Genotoxic Chemical Mutational Studies with a New Program, SnpSift. Front Genet 2012;3:35.

29 Paila U, Chapman BA, Kirchner R, Quinlan AR. GEMINI: integrative exploration of genetic variation and genome annotations. PLoS Comput Biol 2013;9:e1003153.

30 Robinson JT, Thorvaldsdóttir H, Winckler W, Guttman M, Lander ES, Getz G, Mesirov JP. Integrative genomics viewer. Nat Biotechnol 2011;29:24-6.

31 Thorvaldsdottir H, Robinson JT, Mesirov JP. Integrative Genomics Viewer (IGV): high-performance genomics data visualization and exploration. Brief Bioinform 2013:14:178-92.

32 Nur S, Badr R, Sandoval C, Brudniki A, Yeh A. Syndromic presentation of a pleuropulmonary blastoma associated with congenital cystic adenomatoid malformation. A case report. J Pediatr Surg 2007;42:1772-5.
33 Ramasubramanian A, Correa ZM, Augsburger JJ, Sisk RA, Plager DA. Medulloepithelioma in DICER1 syndrome treated with resection. Eye 2013;27:896-7.

34 Anglesio MS, Wang Y, Yang W, Senz J, Wan A, Heravi-Moussavi A, Salamanca C, Maines-Bandiera S, Huntsman DG, Morin GB. Cancer-associated somatic DICER1 hotspot mutations cause defective miRNA processing and reverse-strand expression bias to predominantly mature $3 p$ strands through loss of $5 p$ strand cleavage. J Pathol 2013;229:400-9.

35 Murray MJ, Bailey S, Raby KL, Saini HK, de Kock L, Burke GA, Foulkes WD, Enright AJ, Coleman N, Tischkowitz M. Serum levels of mature microRNAs in DICER1-mutated pleuropulmonary blastoma. Oncogenesis 2014;3:e87.

36 Pugh TJ, Yu W, Yang J, Field AL, Ambrogio L, Carter SL, Cibulskis K, Giannikopoulos P, Kiezun A, Kim J, McKenna A, Nickerson E, Getz G, Hoffher S, Messinger YH, Dehner LP, Roberts CWM, Rodriguez-Galindo C, Williams GM, Rossi $\mathrm{CT}$, Meyerson M, Hill DA. Exome sequencing of pleuropulmonary blastoma reveals frequent biallelic loss of TP53 and two hits in DICER1 resulting in retention of 5p-derived miRNA hairpin loop sequences. Oncogene 2014;33:5295-302.

37 Behjati S, Maschietto M, Williams RD, Side L, Hubank M, West R, Pearson K, Sebire N, Tarpey P, Futreal A, Brooks T, Stratton MR, Anderson J. A pathogenic mosaic TP53 mutation in two germ layers detected by next generation sequencing. PLoS ONE 2014;9:e96531.

38 Foulkes WD, Bahubeshi A, Hamel N, Pasini B, Asioli S, Baynam G, Choong CS, Charles A, Frieder RP, Dishop MK, Graf N, Ekim M, Bouron-Dal Soglio D, Arseneau J, Young RH, Sabbaghian N, Srivastava A, Tischkowitz MD, Priest JR. Extending the phenotypes associated with DICER1 mutations. Hum Mutat 2011;32: $1381-4$.

39 Torrezan GT, Ferreira EN, Nakahata AM, Barros BD, Castro MT, Correa BR, Krepisch AC, Olivieri EH, Cunha IW, Tabori U, Grundy PE, Costa CM, de Camargo B, Galante PA, Carraro DM. Recurrent somatic mutation in DROSHA induces microRNA profile changes in Wilms tumour. Nat Commun 2014;5:4039.

40 Bahubeshi A, Bal N, Rio Frio T, Hamel N, Pouchet C, Yilmaz A, Bouron-Dal Soglio D, Williams GM, Tischkowitz M, Priest JR, Foulkes WD. Germline DICER1 mutations and familial cystic nephroma. J Med Genet 2010;47:863-6.

41 Wu MK, Sabbaghian N, Xu B, Addidou-Kalucki S, Bernard C, Zou D, Reeve AE, Eccles MR, Cole C, Choong CS, Charles A, Tan TY, Iglesias DM, Goodyer PR, Foulkes WD. Biallelic DICER1 mutations occur in Wilms tumours. J Pathol 2013;230:154-64.

42 Takeshita D, Zenno S, Lee WC, Nagata K, Saigo K, Tanokura M. Homodimeric structure and double-stranded RNA cleavage activity of the C-terminal RNase III domain of human dicer. J Mol Biol 2007;374:106-20.

43 Heravi-Moussavi A, Anglesio MS, Cheng S-W, Senz J, Yang W, Prentice L, Fejes AP, Chow C, Tone A, Kalloger SE, Hamel N, Roth A, Ha G, Wan AN, Maines-Bandiera S, Salamanca C, Pasini B, Clarke BA, Lee AF, Lee C-H, Zhao C, Young RH, Aparicio $S A$, Sorensen PH, Woo MM, Boyd N, Jones SJ, Hirst M, Marra MA, Gilks B, Shah $S P$, Foulkes WD, Morin GB, Huntsman DG. Recurrent somatic DICER1 mutations in nonepithelial ovarian cancers. N Engl J Med 2012;366:234-42.

44 Evans DG. Neurofibromatosis type 2 (NF2): a clinical and molecular review. Orphanet J Rare Dis 2009:4:16.

45 Hes FJ, Nielsen M, Bik EC, Konvalinka D, Wijnen JT, Bakker E, Vasen HF, Breuning $\mathrm{MH}$, Tops CM. Somatic APC mosaicism: an underestimated cause of polyposis coli. Gut 2008:57:71-6.

46 Consoli C, Moss C, Green S, Balderson D, Cooper DN, Upadhyaya M. Gonosomal mosaicism for a nonsense mutation (R1947X) in the NF1 gene in segmental neurofibromatosis type 1. J Invest Dermatol 2005;125:463-6.

47 Kinde I, WU J, Papadopoulos N, Kinzler KW, Vogelstein B. Detection and quantification of rare mutations with massively parallel sequencing. Proc Natl Acad SCi USA 2011;108:9530-5.

48 Hiatt JB, Pritchard CC, Salipante SJ, O'Roak BJ, Shendure J. Single molecule molecular inversion probes for targeted, high-accuracy detection of low-frequency variation. Genome Res 2013:23:843-54.

49 Acuna-Hidalgo R, Bo T, Kwint MP, van de Vorst M, Pinelli M, Veltman JA, Hoischen A, Vissers LE, Gilissen C. Post-zygotic point mutations are an underrecognized source of de novo genomic variation. Am J Hum Genet 2015;97:67-74. 